\section{COPD: Risiko bei nicht diagnostizierter Erkrankung stark erhöht}

Çolak Y et al. Prognosis of asymptomatic and symptomatic, undiagnosed COPD in the general population in Denmark: a prospective cohort study. Lancet Respir Med 2017; 5: 426-434

Die chronisch obstruktive Lungenerkrankung (COPD) ist weltweit eine der führenden Krankheits- und Todesursachen. Sie kann frühzeitig mittels Spirometrie diagnostiziert werden, doch Spirometrie wird nur bei symptomatischen Rauchern empfohlen, obwohl frühe Stadien von COPD asymptomatisch sein können. Dänische Forscher untersuchten Personen mit asymptomatischer und symptomatischer, nicht diagnostizierter COPD und bestimmten deren Risiken und Prognosen.

Im Rahmen einer prospektiven Kohortenstudie analysierten Yunus Colak aus Kopenhagen, Dänemark, und Kollegen Daten von Personen aus der Kopenhagener Allgemeinbevölkerungsstudie und bestimmten deren COPD-Risiko. Als Personen mit hohem Risiko nahmen sie nur solche in ihre Studie auf, die im Alter von 40 Jahren oder älter waren, einen kumulativen Tabakkonsum von zehn Packungsjahren oder mehr aufwiesen und bei denen bisher keine Asthmadiagnose vorlag. Die Lungenfunktion maßen sie bei allen Teilnehmern mittels Spirometrie. Sie bestimmten nur die präbronchodilatatorischen Werte von $\mathrm{FEV}_{1}$ und forcierter Vitalkapazität (FVC).
COPD war definiert als $\mathrm{FEV}_{1} / \mathrm{FVC}$ von weniger als $70 \%$ und weniger als die untere Normgrenze sowie $\mathrm{FEV}_{1}$ von weniger als $80 \%$ des vorhergesagten Normwerts. Die Forscher betrachteten Patienten als nicht diagnostiziert, wenn weder ein früherer Krankenhauskontakt wegen COPD noch eine medizinische Behandlung auf COPD registriert waren.

Informationen über Exazerbationen und Pneumonie entnahmen sie dem "National Danish Patient Registry“, den Vitalstatus dem "National Danish Civil Registration System" und die Todesursachen dem "National Danish Causes of Death Registry“. Die Forscher nutzten Cox-Proportional-Hazard-Modelle, um das Risiko von Exazerbationen, Pneumonie, und Todesfällen aufgrund von Atemwegserkrankungen sowie von Todesfällen durch alle Ursachen zu bewerten.

\section{Ergebnisse}

Von insgesamt 95288 gescreenten Personen wiesen 32518 (34\%) ein hohes COPD-Risiko auf. Bei 3699 (11\%) davon trafen die definierten COPD-Kriterien zu. 2903 (78\%) waren nicht diagnostiziert und von diesen wiederum 2052 (71\%) symptomatisch und 851 asymptomatisch. Während einer mittleren Nachbeobachtungszeit von 6,1 Jahren beobachteten die Untersucher bei den 32518 Patienten mit hohem COPD-Risiko 800 Exazerbationen, 2038 Fälle von Pneumonie und 2789 Todesfälle, darunter 152 Todesfälle durch Atemwegserkrankungen.

Im Vergleich zu Personen ohne COPD betrug das auf Alter und Geschlecht adjustierte Wahrscheinlichkeitsverhältnis (Hazard Ratio; HR) für Exazerbationen
5,0, für Pneumonie 1,7, für Tod durch Atemwegserkrankungen 0,7 und für Tod durch alle Ursachen bei Personen mit nicht diagnostizierter, asymptomatischer COPD 1,3. Die entsprechenden HR-Werte bei Personen mit nicht diagnostizierter, symptomatischer COPD waren für Exazerbationen 15,5, für Pneumonie 2,8, für Tod aufgrund von Atemwegserkrankungen 4,3 und für Tod durch alle Ursachen 2,0.

\section{FAZIT}

Nach dem Kenntnisstand der Autoren ist dies die erste Studie mit Fokus auf die Bedeutung von Atemwegssymptomen hinsichtlich der Prognose von Patienten mit nicht diagnostizierter COPD in der allgemeinen Bevölkerung. Personen mit nicht diagnostizierter, symptomatischer COPD hatten ein erhöhtes Risiko von Exazerbationen, Lungenentzündung und Tod. Hingegen lag bei Personen mit nicht diagnostizierter, asymptomatischer COPD ein erhöhtes Risiko von Exazerbationen und Lungenentzündung vor. Diese Ergebnisse deuten darauf hin, dass bessere Initiativen zur Früherkennung und Behandlung von COPD erforderlich sind.

Dr. Volker Kriegeskorte, Untermeitingen 